03

\title{
Анализ пороговых параметров начала акустической кавитации жидкости в зависимости от частоты ультразвукового поля, гидростатического давления и температуры
}

\author{
(С) И.В. Смирнов, Н.В. Михайлова, Б.А. Якупов, Г.А. Волков
}

Санкт-Петербургский государственный университет, 199034 Санкт Петербург, Россия e-mail: ivansmirnov.sci@gmail.com

Поступило в Редакцию 29 декабря 2020 г. В окончательной редакции 1 апреля 2021 г. Принято к публикации 15 мая 2021 г.

\begin{abstract}
Проведено сравнение критерия инкубационного времени кавитации и классического критерия нестабильной кавитации. Показано, что критерий инкубационного времени кавитации может быть универсальной основой для оценки пороговых значений отрицательного давления в широком диапазоне частот колебаний звукового поля, при различных температурах жидкости и значениях гидростатического давления. В отличие от классического критерия он не требует информации о микропараметрах дефектной структуры материала. С другой стороны, комбинация двух критериев позволяет определить частотную зависимость диапазона радиусов активных кавитационных зародышей. Кроме того, обсуждена физическая сущность макропараметров жидкости, используемых в критерии инкубационного времени кавитации. Показано, что инкубационное время кавитации может быть связано с числом Гиббса и скоростью нуклеации зародышей кавитации.
\end{abstract}

Ключевые слова: акустическая кавитация, ультразвук, пороговая амплитуда, критерий инкубационного времени.

DOI: 10.21883/JTF.2021.11.51521.352-20

\section{Введение}

Акустической кавитацией называется нарушение сплошности жидкости или развитие существующих в ней микропузырьков в результате локального снижения давления при прохождении акустической волны большой интенсивности. Кавитационные пузырьки, заполненные парогазовой смесью, образуются в фазе разрежения акустической волны, когда амплитуда звукового давления превышает определенное пороговое значение. Относительно дальнейшей динамики развития микропузырька принято отделять две стадии акустической кавитации. В случае инерционной (нестабильной) кавитации после наступления фазы сжатия акустической волны кавитационные пузырьки схлопываются. Это приводит к локальному разогреву и гидродинамическим возмущениям в жидкости, например, кумулятивным струям и локальным ударным волнам. В случае неинерционной (стабильной) кавитации пузырьки пульсируют на протяжении многих периодов смены фаз растяжения и сжатия без схлопывания. Данная стадия кавитации приводит к физическим процессам с меньшей плотностью энергии, например, акустическим микротечениям. В общем случае обе стадии конкурируют между собой и зависят от текущей комбинации частоты и интенсивности звуковых колебаний, размеров начальных зародышей кавитации, параметров и температуры жидкости, а также гидростатического давления. Детальный анализ процессов акустической кавитации можно найти в классических работах $[1,2]$.

Благодаря физическим и химическим эффектам при акустической кавитации она активно применяется как в фундаментальных, так и прикладных исследованиях. Тематика актуальных исследований с использованием акустической кавитации довольно обширна. Например, обработка пищевых продуктов [3,4], процессы в сонохимии $[5,6]$, воздействие на микроорганизмы и биологические вещества $[7,8]$, литье металлов $[9,10]$, медицина $[11,12]$, фазовые переходы [13-15], а также продолжение исследования самих процессов кавитации $[16,17]$. Анализ процессов акустической кавитации обычно проводится на основе описания динамики кавитационных пузырьков и оценки порога кавитации для заданных внешних условий, параметров звукового воздействия и параметров жидкости $[1,2,18,19]$. Несмотря на интенсивные исследования теоретических подходов к анализу кавитации, многие вопросы прогноза порога кавитации остаются нерешенными. Однако для успешного применения акустической кавитации в актуальных исследованиях необходимо уметь определять ее пороговые характеристики. Например, хорошо известно, что порог начала акустической кавитации зависит от частоты ультразвука или длительности импульсного акустического воздействия [1]. Однако классические уравнения для оценки порога кавитации $[18,19]$ не учитывают временной фактор. Это усложняет оценку частотной зависимости порога кавитации. Еще одна особенность класси- 
ческого подхода к оценке порога кавитации связана с включением в его уравнение микропараметра - радиуса кавитационного зародыша. Такой подход безусловно оправдан. Однако он требует знать заранее размеры и наполнение дефектов в жидкости, что, в общем случае, довольно затруднительно. Для практического применения акустической кавитации более приемлемо использовать макропараметры жидкости, которые доступны в справочных источниках, а также могут быть определены прямым расчетом или экспериментом.

В работах [20-22] был предложен подход, который позволяет подойти к оценке пороговых характеристик акустической кавитации с новых позиций. Поскольку кавитация это динамический процесс, необходимо учитывать соотношение характерного времени протекания переходных процессов в жидкости с длительностью воздействия. Согласно классическому подходу к оценке кавитационной прочности жидкости, зародыш кавитации начнет расширяться, если локальное отрицательное давление, вызванное звуковой волной, достигнет некоторой критической величины. Однако в таком случае при воздействии сколь угодно малой длительности нарушается закон сохранения импульса. Поэтому достичь только некоторой критической величины отрицательного давления недостаточно. Необходимо чтобы критической величины достиг и приложенный импульс. На этой концепции основан критерий инкубационного времени разрушения, который был успешно применен для ряда задач динамического разрушения и текучести твердых тел [21,23-26]. Наблюдаемая аналогия между динамическими процессами разрушения сплошной среды позволила предложить критерий инкубационного времени кавитации [20-22]. Предложенный критерий дает возможность учитывать не только влияние частоты ультразвука, но и температуры жидкости [27]. Тем нем менее данный критерий рассматривался независимо от классических подходов к оценке порога кавитации жидкости. Отсюда возникает вопрос о сравнении предсказательной способности критерия инкубационного времени кавитации с параметрами классического критерия порога кавитации. Поскольку критерий инкубационного времени предполагает использование только макропараметров жидкости, такое сравнение может помочь связать характеристики жидкости и процессов, протекающих на микроуровне, с макроскопическими характеристиками прочности жидкости.

В настоящей работе проводится анализ пороговых характеристик начала акустической кавитации жидкости на примере воды в зависимости от частоты ультразвука, гидростатического давления и температуры. Анализ основан на сравнении расчетов по критерию инкубационного времени кавитации [27] и критерию порога нестабильной кавитации [19]. Результаты численных расчетов сравниваются с экспериментальными данными из литературы. Внимание работы сосредоточено на возможности использования в рамках подхода инкубационного времени только макропараметров жидкости для прогноза начала нестабильной кавитации при различных внешних условиях. Кроме того, рассматривается возможность комбинации нового и классического подходов при необходимости учета конкретных параметров дефектной структуры воды.

\section{1. Теоретические подходы к оценке кавитационной прочности жидкости}

Анализ кавитационной прочности жидкости может проводиться с помощью простых нелинейных дифференциальных уравнений движения стенки пузыря, например, уравнения Нолтинга-Непайраса $[1,28]$ или уравнения Херринга-Флинна [1,2]. Решения этих уравнений показывают, что пороговое давление кавитации зависит от ряда параметров, таких как гидростатическое давление, радиус зародыша кавитации, частота и амплитуда ультразвуковых колебаний, а также температура жидкости. Рассмотрим два подхода к оценке кавитационной прочности жидкости. Первый подход основан на учете параметров микропузырьков, которые содержатся в жидкости в большом количестве и разнообразии. Второй подход основан на введении макропараметров прочности жидкости. Применение и сравнение этих подходов будет рассмотрено в разд. 2.

\section{1. Пороговые характеристики кавитационных пузырьков}

Кавитационные пузырьки в жидкости возникают в ультразвуковом поле на зародышах, которые представляют собой паровые, газовые или твердые микронеоднородности. В результате воздействия ультразвука кавитационные полости наполняются газом, растворенным в жидкости, и/или паром до некоторого состояния с равновесным радиусом $R_{0}$. Рост парогазового пузырька ограничен гидростатическим давлением, силой поверхностного натяжения и присоединенной массой жидкости. Такие пузырьки стабильно существуют в ультразвуковом поле. При превышении амплитуды акустических колебаний в фазу отрицательного давления некоторой критической величины микропузырек начинает расти до некоторого максимального радиуса, а в положительный полупериод схлопывается. Соответствующую критическую величину отрицательного давления принимают за кавитационную прочность жидкости.

При воздействии ультразвука на кавитационный пузырек давление на бесконечности $P_{\infty}$ можно представить в виде

$$
P_{\infty}=P_{s t}-A \sin \omega t,
$$

где $a$ - амплитуда давления ультразвукового поля, $\omega=2 \pi f-$ угловая частота при частоте ультразвуковых колебаний $f, P_{s t}$ - гидростатическое давление.

В работах $[1,19]$ было показано, что для начальной стадии кавитации выражение для прочности жидкости на 
пузырьке радиуса $R_{0}$ может быть представлено в виде

$$
P_{t h}=P_{s t}+\frac{4}{3 \sqrt{3}} \frac{\sigma}{R_{0}} \sqrt{\frac{2 \sigma}{P_{s t} R_{0}+2 \sigma}}
$$

где $\sigma$ - поверхностное натяжение для данной жидкости, $R_{0}$ - начальный радиус пузырька. Таким образом, пузырек устойчив пока величина $A \sin \omega t$ не достигнет $P_{t h}$ в противном случае пузырек начинает расти, т.е. разрывается. Отметим, что выражение (2) было получено при условии, что давление насыщенных паров в жидкости много меньше гидростатического давления, а диффузией газа можно пренебречь. Кроме того, в общем случае в жидкости при воздействии акустической волны возникает одновременно множество пузырьков. Однако на начальной стадии кавитации пузырьки можно рассматривать как одиночные, пренебрегая их взаимодействием $[1,19]$. Это дает возможность проводить анализ только для одного пузырька. При более поздних стадиях кавитационного процесса указанные упрощения могут быть некорректны $[1,19]$. В настоящей работе рассматривается только начало кавитации.

В выражении (2) не учитывается частота ультразвукового воздействия. При этом экспериментально показано $[1,29]$, что кавитационная прочность зависит от частоты ультразвука. Связь частоты ультразвуковых колебаний и кавитации может быть получена на основе выражения для резонансного радиуса пузырька $R_{r}[1,2,30]$ :

$$
f_{r}=\frac{2 \pi R_{r}}{\frac{3 \gamma}{\rho}\left(P_{s t}+\frac{2 \sigma}{R_{r}}\right)},
$$

где $f$ - резонансная частота пузырька радиуса $R_{r}$, $\gamma$ - показатель политропы, характеризующий уравнение состояния парогазовой смеси в пузырьке, $\rho$ плотность жидкости. Представленное выражение может быть использовано только для гармонических колебаний. В случае частоты ультразвукового поля $f$ выше резонансной частоты $f_{r}$ рост и схлопывание пузырька с радиусом $R_{0} \geq R_{r}$ не будет происходить в один период колебаний. Такой случай кавитации в настоящей работе не рассматривается.

Выражения (2) и (3) определяют диапазон радиусов пузырьков, которые могут участвовать в процессе начала кавитации. Согласно (2), при достижении пороговой амплитуды колебаний $P_{t h}$ для пузырька зародыша кавитации с радиусом $R_{0}$ все зародыши кавитации с радиусом более $R_{0}$ также должны приводить к кавитации. С другой стороны, согласно (3), для заданной частоты ультразвука существует радиус $R_{r}$, который ограничивает максимальный размер кавитирующих зародышей. На рис. 1 приведены примеры с оценкой кавитационной прочности воды и резонансной частоты начального пузырька в зависимости от его радиуса $R_{0}$ при фиксированных значениях $\sigma=0.073 \mathrm{~N} / \mathrm{m}, P_{s t}=100 \mathrm{kPa}, \gamma=1.3, \rho=1000 \mathrm{~kg} / \mathrm{m}^{3}$. Диапазон активных кавитационных зародышей $\Delta R_{a c}$ обусловлен минимальным кавитационным зародышем

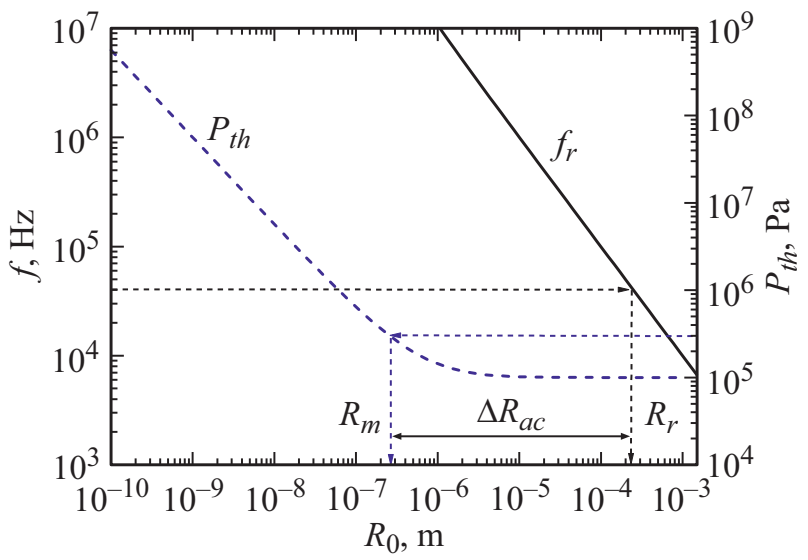

Рис. 1. Расчетные значения кавитационной прочности воды $P_{t h}$ и резонансной частоты $f_{r}$ начального пузырька для различных значений радиуса начального пузырька $R_{0}$. Пояснения в тексте.

$R_{m}$, который зависит от пороговой амплитуды колебаний, а также максимальным кавитационным зародышем $R_{r}$, который имеет резонансную частоту, равную частоте звуковых колебаний. Пример оценки диапазона активных кавитационных зародышей схематично представлен на рис. 1.

Нужно отметить, что на результат расчетов в общем случае может влиять кинетическая энергия присоединенной массы жидкости. В работе [1] было показано, что для случая воды и отсутствия в ней пузырьков с радиусом более $10^{-5} \mathrm{~m}$ учетом инерции воды можно пренебречь. Поэтому в настоящей работе для простоты изложения инерция жидкости не учитывается. Тем не менее в развитие представленных ниже результатов для более корректной оценки отрицательного давления в области кавитации можно обратиться к работам $[1,2,31,32]$.

\section{2. Подход инкубационного времени кавитации}

Увеличение частоты ультразвука приводит к уменьшению периода отрицательного давления. Следовательно, возрастание порога кавитационной прочности жидкости при увеличении частоты ультразвука сопровождается уменьшением длительности и увеличением скорости растягивающего воздействия на пузырек. Аналогичный эффект наблюдается при высокоскоростном или интенсивном кратковременном нагружении твердого тела, когда с увеличением скорости деформации увеличивается критическое напряжение разрушения. Кавитация, как и динамическое разрушение твердых тел, это процесс, протекающий во времени, а для ее возникновения необходимо подвести в зону разрушения определенное количество энергии. Достичь критического давления уже недостаточно, необходимо выдержать его превышение в течение определенного времени. Такая аналогия позволила предложить критериальное условие для начала 
кавитации на основе подхода инкубационного времени разрушения [20-22,33].

В общем виде критерий инкубационного времени кавитации может быть представлен $[27,34]$ как

$$
\frac{1}{\tau(T)} \int_{t=\tau(T)}^{t} \operatorname{sign}\left(P\left(t^{\prime}\right)\right)\left|\frac{P\left(t^{\prime}\right)}{P_{c}(T)}\right|^{\alpha} d t^{\prime} \geq 1,
$$

где $P(t)$ - изменение локального растягивающего (отрицательного) давления, $P_{c}-$ порог кавитации при низких частотах (когда изменение частоты не приводит к существенному изменению порога кавитации), $T$ температура жидкости, $\alpha$ - параметр, характеризующий чувствительность жидкости к истории нагружения, $\tau$ - инкубационное время кавитации, которое характеризует развитие зародыша кавитации на временной шкале. При выполнении критерия (4) звуковое поле будет инициировать рост пузырьков. Начало кавитации осуществляется в первый момент времени $t$, когда выполнилось условие (4). При этом $P_{c}$ и $\tau$ соответствуют некоторому представительному объему, который определяет масштабный уровень рассмотрения процесса кавитации в результате действия звукового поля.

Поскольку температура жидкости влияет на низкочастотный порог кавитации $P_{c}$, то его значение можно определить по формуле

$$
P_{c}(T)=P_{s t}-P_{p h}(T),
$$

где $P_{s t}$ - гидростатическое давление, а $P_{p h}(T)$ - давление, описываемое фазовой диаграммой „жидкость-пар“. Согласно термодинамической диаграмме фазового перехода, повышение температуры может инициировать механизм фазового перехода, известный как кипение. Снижение статического давления также может инициировать другой механизм фазового перехода, известный как кавитация. Таким образом, кавитация определяется как процесс образования паровой фазы жидкости, когда она подвержена снижению давления при постоянной внешней температуре. Поэтому кавитация произойдет только в том случае, если локальное давление упадет до некоторой величины, ниже давления насыщенного пара жидкости.

Согласно концепции инкубационного времени [21,27], значения $\tau$ определяются скоростью переходных процессов изменения структуры среды. По аналогии с теорией термодинамических флуктуаций температурную зависимость инкубационного времени кавитации можно определить следующим выражением:

$$
\tau(T)=\tau_{0} e^{\frac{G}{k T}}
$$

где $\tau_{0}-$ инкубационное время, характерное для данного масштаба, $k-$ постоянная Больцмана $\left(k=1.3807 \cdot 10^{-23} \mathrm{~J} / \mathrm{K}\right), G$ - доля энергии, необходимая для начала кавитации в представительном объеме заданного масштабного уровня.
Звуковое давление описывается выражением

$$
P(t)=A \sin \omega t
$$

Подстановка выражений (5)-(7) в критерий (4) дает выражение для расчета порогового давления начала кавитации жидкости [27]:

$$
P_{t h}(T)=\left\{\begin{array}{c}
\left.\left.P_{c}(T)\left|\frac{1}{\lambda(T)} \max _{t_{z} \in(0 ; \pi)} \int_{t_{z}-\lambda(T)}^{t_{z}}\right| \sin z\right|^{\alpha} d z\right|^{-\frac{1}{\alpha}}, \\
\lambda(T) \leq \pi, \\
\left.\left.P_{c}(T)\left|\frac{1}{\lambda(T)} \int_{0}^{\pi}\right| \sin z\right|^{\alpha} d z\right|^{-\frac{1}{\alpha}}, \\
\lambda(T)>\pi,
\end{array}\right.
$$

где $\lambda(T)=\tau(T) \omega$ и $z=\omega t$, а $t \in(0, \pi / \omega)$.

Выражение (8) позволяет оценить порог давления для начальной стадии кавитации для различных частот ультразвукового воздействия, при разных температурах жидкости и гидростатическом давлении. При этом в нем используются только макропараметры жидкости. Обсуждение способов определения этих параметров будет проведено в следующем разделе. Отметим, что критерий инкубационного времени кавитации хорошо показал себя при моделировании порога акустической кавитации морской воды в диапазоне частот акустических колебаний $2-15 \mathrm{kHz}$ [33], а также при моделировании зависимости порога кавитации воды от длительности ударной волны [20].

\section{2. Расчеты пороговых характеристик начала акустической кавитации жидкости и их анализ}

\section{1. Частотная зависимость порога кавитации}

Рассмотрим результаты расчетов зависимости порогового давления для начала кавитации от частоты ультразвукового воздействия по критерию (4) на примере воды (рис. 2). Теоретическая зависимость 3 на рис. 2 была получена с помощью численных расчетов при $\tau=3.3 \cdot 10^{-7} \mathrm{~s}, \alpha=0.5, P_{s t}=1 \mathrm{~atm}=1.01 \cdot 10^{5} \mathrm{~Pa}$. Для сравнения добавлены данные из работ $[35,36]$. Результаты расчетов качественно описывают частотную зависимость порога акустической кавитации.

К сожалению, экспериментальные исследования зависимости порога кавитации от частоты ультразвука мало представлены в литературе. Кроме того, такие исследования не могут быть реализованы, по крайней мере, для существующего уровня техники, на одном и том же оборудовании в широком диапазоне частот звуковых колебаний. Поэтому сопоставление результатов обычно проводится либо по экспериментам с различной фиксированной частотой ультразвука, либо по результатам 


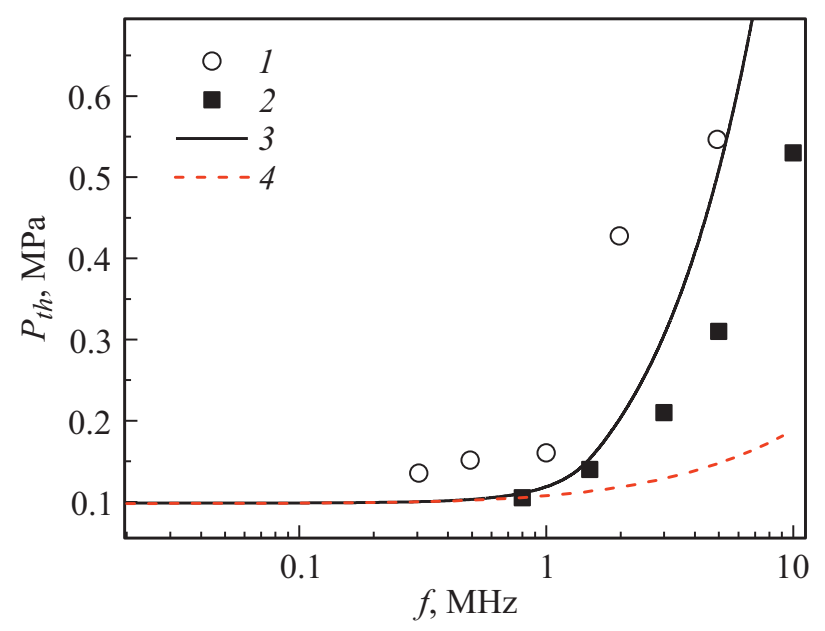

Рис. 2. Пороговое отрицательное давление для начала кавитации $P_{t h}$ в зависимости от частоты ультразвука $f .1$ - данные из работы [36]; 2 - данные из работы [35]; 3 - расчет по критерию инкубационного времени кавитации (4); 4 - расчет по формуле (2) для резонансного пузырька, рассчитанного по формуле (3).

численного моделирования, которые апробированы только для конкретной частоты ультразвука. В обоих случаях могут быть получены качественные зависимости исследуемых характеристик, но корректность количественной точности анализа может подвергаться сомнениям. Решение этой проблемы возможно с помощью постановки специальных экспериментов, где необходимые изменения технических решений экспериментальной установки будут сведены к минимуму, а также будут минимизированы погрешности, связанные с измерением параметров воздействия на жидкость и параметров кавитации. Другое решение проблемы - точности моделей может быть получено с помощью развития методов компьютерного моделирования [37-41], в том числе с применением методов машинного обучения.

На рис. 2 приведена зависимость порога акустической кавитации для пузырька, у которого резонансная частота равна частоте ультразвука (кривая 4). Расчеты проводились для $\sigma=0.073 \mathrm{~N} / \mathrm{m}, P_{s t}=100 \mathrm{kPa}, \gamma=4 / 3$, $\rho=1000 \mathrm{~kg} / \mathrm{m}^{3}$. Как было отмечено выше (см. разд. 1.1 ), это условие ограничивает максимальный размер зародыша, который может быть задействован в процессе начала кавитации при пороговом давлении. Данная зависимость была получена с помощью подстановки (2) в (3). Из рис. 2 видно, что порог кавитации резонансного микропузырька близок к порогу кавитации, рассчитанному по выражению (8), примерно до частоты $500 \mathrm{kHz}$. Согласно примеру на рис. 1 , достижение порогового давления кавитации, рассчитанного по формуле (2), приводит к началу кавитации большого диапазона зародышей $\Delta R_{a c}$. При этом заметим, что достигнутое минимальное пороговое давление кавитации является пороговым для всех размеров зародышей из диапазона $\Delta R_{a c}$. Дальнейшее повышение порогового отрицатель- ного давления связано с уменьшением минимального радиуса зародыша кавитации, но оно уже является запороговым давлением для зародышей с радиусом более минимального $R_{m}$, вплоть до резонансного радиуса зародыша $R_{r}$. В свою очередь повышение частоты ультразвука приводит к снижению резонансного радиуса пузырька. Этим можно объяснить продолжительный участок почти постоянного порогового давления кавитации в диапазоне частот ультразвука до $500 \mathrm{kHz}$ на рис. 2. Несмотря на возможное отсутствие в жидкости зародышей кавитации с резонансными радиусами, которые соответствуют частотам ультразвука до $500 \mathrm{kHz}$ (на рис. 1 соответствует $\left.R_{r} \approx 20 \mu \mathrm{m}\right)$, расчетное давление их активации совпадает с пороговым отрицательным давлением для зародышей с меньшими радиусами. Начиная с определенной частоты ( $\approx 500 \mathrm{kHz}$ для рассматриваемого примера), размер резонансного зародыша становится настолько мал, что пороговое давление для его активации начинает существенно возрастать.

Таким образом, при увеличении частоты ультразвука происходит сдвиг диапазона радиуса активных зародышей $\Delta R_{a c}$ в сторону меньших размеров. Однако возникает вопрос, как меняется сам диапазон размеров зародышей, которые могут привести к кавитации жидкости при заданной комбинации частоты и амплитуды ультразвука (при условии достижения отрицательным давлением порогового значения при соответствующей частоте ультразвука).

Предположим, что пороговое давление (2) равно пороговому давлению кавитации (8). Тогда, учитывая фазовое состояние жидкости при температуре $293 \mathrm{~K}$, приравняем (2) и (8) и построим зависимость между минимальным радиусом зародыша кавитации и частотой ультразвука. На рис. 3 представлены результаты вычисления такой зависимости. Условия расчетов были

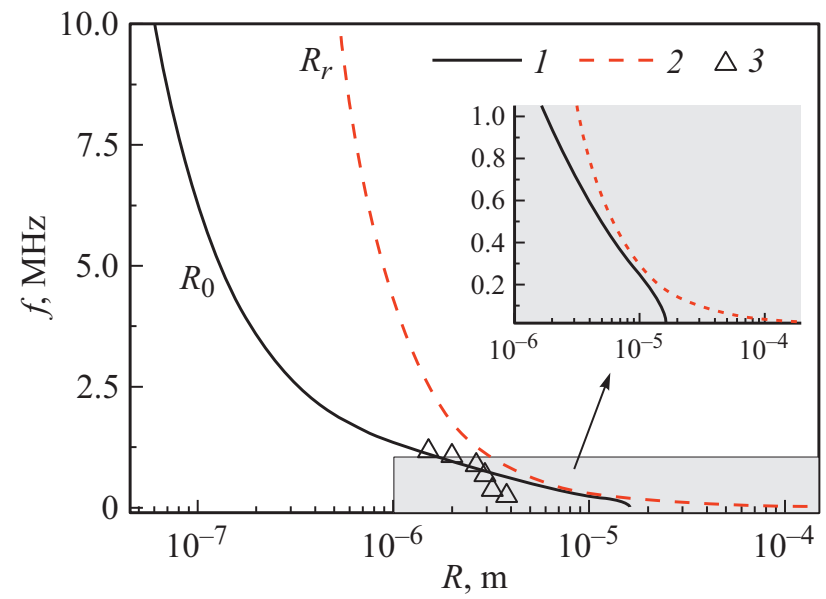

Рис. 3. Зависимость между радиусами зародышей, которые могут участвовать в процессе начала кавитации, и частотой ультразвука. 1 - расчет с применением критерия инкубационного времени кавитации (4); 2 - расчет по формуле (3) для радиуса пузырька $R_{r}$ с резонансной частотой, равной частоте ультразвука; 3 - данные из работы [42]. 
те же, что даны для рис. 2. На рис. 3 видно, что минимальный размер возможного зародыша кавитации в воде (кривая 1 ) при низких частотах до $\approx 50 \mathrm{kHz}$ постоянен, а при дальнейшем увеличении частоты звуковых колебаний начинает существенно меняться. Резонансный (он же максимальный) размер зародыша (кривая 2) быстро уменьшается с увеличением частоты ультразвука до $\approx 300 \mathrm{kHz}$, после чего его уменьшение замедляется. Диапазон размеров возможных активных зародышей кавитации с увеличением частоты ультразвука сначала уменьшается до минимального при $\approx 300 \mathrm{kHz}$, а потом расширяется. Этот переход, по-видимому, приводит к существенному расхождению расчетных кривых после $300-500 \mathrm{kHz}$. Заметим, что в общем случае порог кавитационной прочности жидкости зависит от наибольшего зародыша из всех имеющихся в диапазоне $\Delta R_{a c}$ в озвучиваемом объеме жидкости.

На рис. 3 добавлены результаты из работы [42], где было построено распределение кавитационных пузырьков по размеру на разных частотах ультразвука с помощью определения интенсивности сонохемилюминесценции в дистиллированной воде с люминолом. В указанной работе отмечалось существенное отличие расчетных и наблюдаемых размеров кавитационных пузырьков. Расчеты на рис. 3 показывают, что реальные размеры зародышей могут быть предсказаны с помощью предложенной модели. Варьируя частоту и амплитуду ультразвука, можно подобрать оптимальный режим для начала кавитации жидкости, в том числе с учетом конкретных размеров зародышей.

\section{2. Зависимость порога начала кавитации от температуры и гидростатического давления}

Помимо частотной зависимости, порог начала кавитации имеет существенную зависимость от температуры жидкости и гидростатического давления. Выражение (8) позволяет рассчитать такие зависимости. На рис. 4 представлены результаты моделирования температурной зависимости пороговой амплитуды кавитации. Расчеты проводились для фиксированной частоты ультразвуковых колебаний в жидкости $f=25.5 \mathrm{kHz} \tau_{0}=7 \cdot 10^{-9} \mathrm{~s}$ и $\alpha=0.5$. Результаты моделирования сравниваются с экспериментальными данными [43], полученными при тех же условиях. Учет изменения низкочастотного порога кавитации $P_{c}$ с изменением температуры, согласно фазовой диаграмме „жидкость-пар“ в формуле (5) и влияния температуры на инкубационное время кавитации в формуле (6), позволил обеспечить хорошее соответствие расчетов экспериментальным данным. Качественное совпадение теоретических кривых с экспериментальными данными было получено при $G=3420 \cdot 10^{-23} \mathrm{~J}$ в случае гидростатического давления $P_{s t}=2.5 \mathrm{MPa}$ и $G=3350 \cdot 10^{-23} \mathrm{~J}$ для $P_{s t}=7.5 \mathrm{MPa}$. Повышение внешнего давления $P_{s t}$ привело к небольшому уменьшению
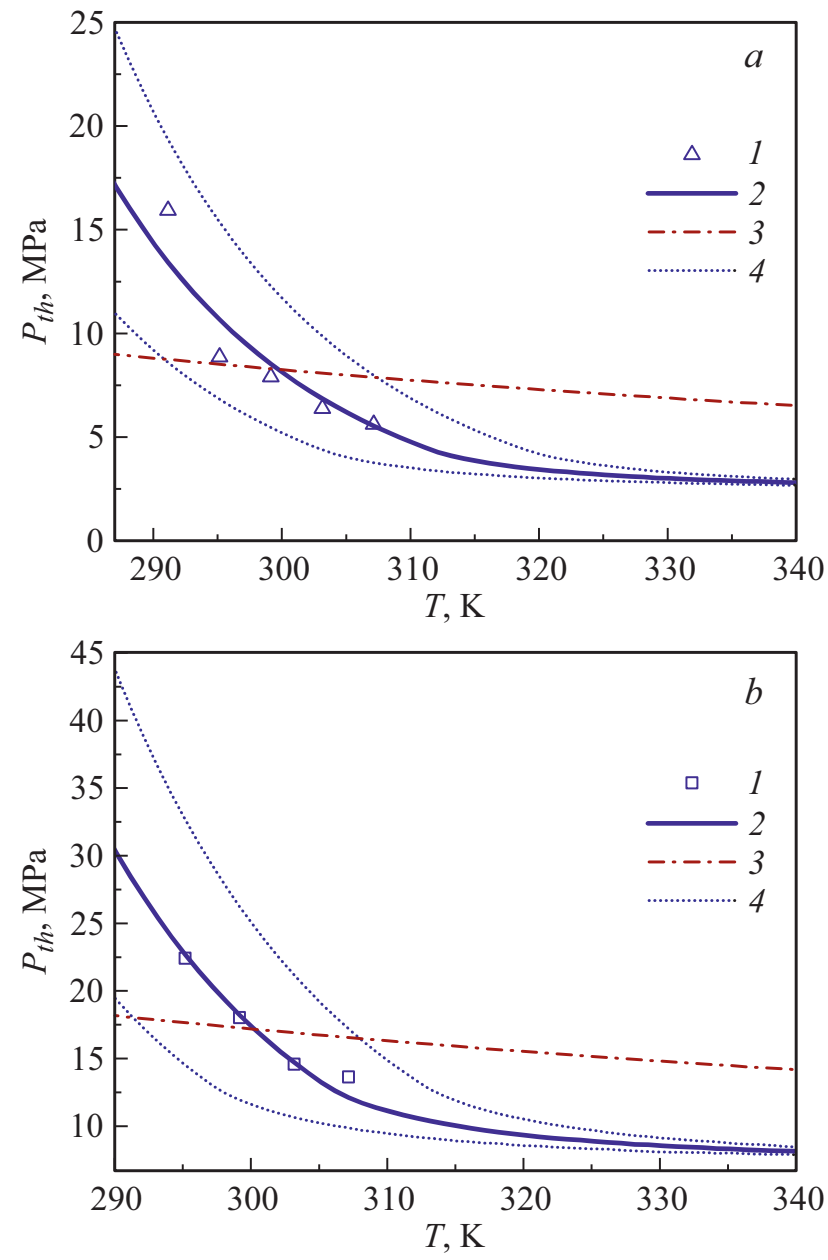

Рис. 4. Зависимость порогового отрицательного давления для начала кавитации $P_{t h}$ в зависимости от температуры жидкости T. $a-P_{s t}=2.5 \mathrm{MPa} ; b-P_{s t}=7.5 \mathrm{MPa}$. $1-$ экспериментальные данные из [43]; 2 - расчет по критерию инкубационного времени кавитации (4); 3 - расчет по формуле (2) с учетом влияния температуры; 4 - диапазон смещения расчетной кривой 2 при вариации $\tau_{0}$ на $\pm 20 \%$.

доли энергии, необходимой для начала кавитации в представительном объеме жидкости. Кроме того, на рис. 4 показано возможное смещение расчетной кривой 1 при вариации $\tau_{0} \pm 20 \%$ (кривые 4 ).

На рис. 4 добавлены зависимости (кривые 3), рассчитанные по классическому критерию (2) с учетом зависимости от температуры радиуса зародыша $R_{0}$ и коэффициента поверхностного натяжения $\sigma$. Для зависимости $R_{0}(T)$ была построена эмпирическая формула по экспериментальным точкам, представленным в работе [1]:

$$
R(T)=R_{a m}\left(\frac{1-a}{T_{a m}^{2}} T^{2}+a\right),
$$

где $R_{a m}-$ радиус пузырька при температуре $T_{a m}=292.15 \mathrm{~K}, a=0.01$. В случае $P_{s t}=2.5 \mathrm{MPa} \mathrm{ради-}$ ус $R_{a m}=8.5 \cdot 10^{-9} \mathrm{~m}$. В случае $P_{s t}=7.5 \mathrm{MPa}$ радиус $R_{a m}=4.5 \cdot 10^{-9} \mathrm{~m}$. Для коэффициента поверхностного 
натяжения использовалось правило Этвеша [44] для воды:

$$
\sigma(T)=0.07275(1-0.002(T-291))
$$

Несмотря на попытку учесть влияние температуры для порога кавитации в классической формуле (2), расчетная кривая 3 на рис. 4 удовлетворяет экспериментальным точкам только в диапазоне температур 295-300 K, для которых она была получена изначально $[1,19]$. В первую очередь это может быть связано с используемыми упрощениями, например, пренебрежением величиной давления внутри пузырька и также диффузионным потоком газа. Коррекция формулы (2) на рассматриваемый случай выходит за рамки настоящей работы. Подробный анализ особенностей формулы (2) при различных внешних условиях и параметрах жидкости можно найти в $[1,2]$. Рассмотренный пример показывает, что в отличие от классической формулы для порога кавитации (2), критерий инкубационного времени кавитации (4) не требует добавлять дополнительные корректировочные параметры. Используемый набор параметров вполне достаточен для построения зависимости порога для начала кавитации от температуры жидкости и гидростатического давления.

В дополнение рассмотрим расчеты по критерию инкубационного времени (4) зависимости порогового давления для начала кавитации от гидростатического давления при фиксированной температуре. Расчеты проводились при фиксированных параметрах $\tau_{0}=7 \cdot 10^{-9} \mathrm{~s}$, $G=3420 \cdot 10^{-23} \mathrm{~J}$, которые были использованы для случая давления $P_{s t}=2.5 \mathrm{MPa}$ на рис. 4. Экспериментальные данные были взяты из работы [43].

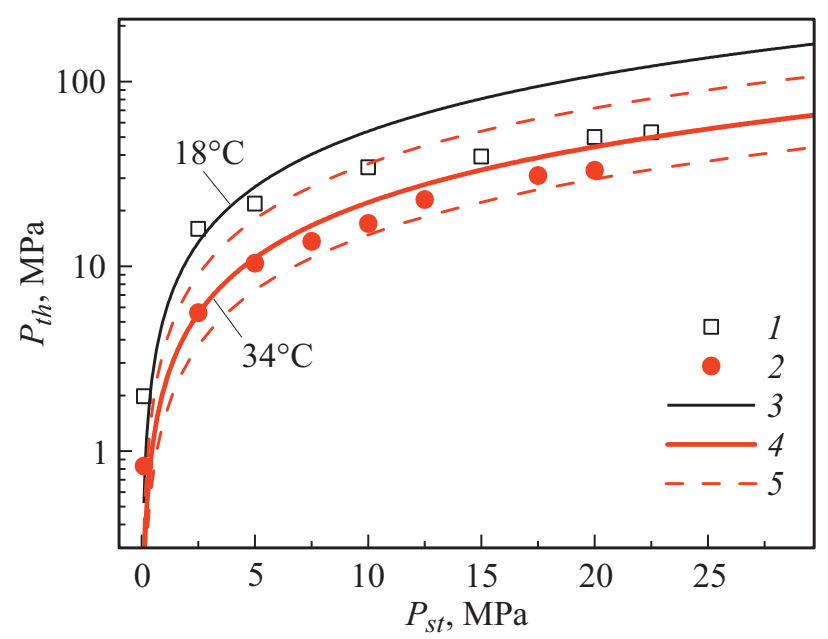

Рис. 5. Зависимость порогового давления для начала кавитации от гидростатического давления при температурах жидкости 18 и $34^{\circ} \mathrm{C}$. Ось ординат в логарифмическом масштабе. 1,2 - экспериментальные точки [43] для температур 18 и $34^{\circ} \mathrm{C}$ соответственно; 3,4 - расчет по критерию инкубационного времени кавитации для температур 18 и $34^{\circ} \mathrm{C}$ соответственно; 5 - диапазон смещения расчетной кривой 4 при вариации параметра $G$ на $\pm 3 \%$.
Согласно расчетам на рис. 4, изменение гидростатического давления приводит к изменению параметра $G$. Рис. 5 подтверждает данное наблюдение. Фиксированное значение $G$ обеспечивает хорошее соответствие расчетов экспериментальным данным только для точек давления до $5 \mathrm{MPa}$. После этого значения давления расчетные кривые порога кавитации идут сильно выше экспериментальных данных. Изменение значения $G$ на $\pm 3 \%$ (кривые 5 на рис. 5) показывает, что требуется постепенное снижение значения параметра $G$ с увеличением гидростатического давления $P_{s t}$. Чтобы установить данную зависимость необходимо провести дополнительные исследования.

\section{3. Замечание о параметрах критерия инкубационного времени кавитации}

Для использования критерия инкубационного времени кавитации необходимо знать макроскопические параметры жидкости $P_{c}, \tau$ и $\alpha$. Кавитационная прочность жидкости $P_{c}$ может быть определена экспериментально при низких частотах колебаний. Однако еще нет данных о прямом определении в эксперименте или с помощью прямых расчетов инкубационного времени кавитации $\tau$ и параметра $\alpha$. Последние два параметра определяются полуэмпирически, путем подбора расчетных кривых с наименьшим отклонением от экспериментальных точек. Тем не менее ряд результатов показывает возможность определения этих параметров с помощью прямого расчета.

В работе [22] было получено, что безразмерный параметр $\alpha$ меняется в зависимости от вязкости жидкости. Для порогового давления в случае кратковременного нагружения длительностью $T$ должно выполняться соотношение $P_{t h}^{\alpha} T=$ const. Хорошее соответствие расчетных зависимостей и экспериментальных данных было получено при $\alpha=0.5$ для воды и $\alpha=1$ для глицерина. При этом показано, что эти значения получаются при учете конкуренции вклада вязкой и инерционной составляющей в уравнении динамики кавитационного пузырька. Таким образом, можно заключить, что параметр $\alpha$ косвенно учитывает вклад вязкости и приведенной массы в динамику кавитационного зародыша. Однако способ определения $\alpha$ еще требует своей более полной экспериментальной проверки.

Инкубационное время кавитации $\tau$ может рассматриваться как независимый параметр жидкости, например, это было сделано в работах [20,22,33] или разд. 2.1 настоящей работы. При рассмотрении температурных задач необходимо учитывать влияние температуры на параметр $\tau$. Такую зависимость можно учесть с помощью выражения (6). Как показано в [27] и в разд. 2.2, введение параметра $\tau_{0}$ и характеристики энергии, необходимой для начала кавитации в некотором представительном объеме жидкости, позволяет моделировать температурную зависимость порога кавитации. До сих пор эти параметры подбирались из условия наложения 
расчетных кривых на экспериментальные точки. Однако обратим внимание, что показатель степени в (6) имеет вид так называемого числа Гиббса $[2,45]$ :

$$
G b=\frac{W_{c r}}{k T},
$$

где $W_{c r}$ - энергия, которая должна быть выделена для образования пузырька. Эта энергия может быть рассчитана, как

$$
W_{c r}=\frac{4}{3} \pi R_{C}^{2} \sigma=\frac{16 \pi \sigma^{3}}{3 \Delta P_{c r}^{2}},
$$

где $R_{c}$ - критический радиус для роста зародыша или критическая вакансия размером в несколько межмолекулярных расстояний, $\Delta P_{c r}-$ прочность жидкости на растяжение. Это одно из основных уравнений теории гомогенной нуклеации. Согласно этой теории [2], энергия для гомогенного зародышеобразования должна достичь величины $W_{c r}$ за определенное время. Тогда выражение (10) соответствует вероятности того, что жидкость сможет выдержать растягивающее давление $\Delta P_{c r}$ в течение данного времени. В изолированной системе задача гомогенного зародышеобразования может сводиться к оценке локального возмущения энергии $W_{c r}$ в результате стохастического движения молекул. Такая связь выражается через кинетическую энергию молекул в выражении (9). Поэтому выражение (10) можно рассматривать, как определенную вероятность зародышеобразования в обусловленном изолированном локальном объеме в течение обусловленного времени.

Рассчитаем размер предполагаемой критической вакансии $R_{c}$ для примера на рис. $4, a$. Согласно расчетам, $W_{c r}=G=3420 \cdot 10^{-23} \mathrm{~J}$. Подставим это значение в формулу (10). Тогда, сделав соответствующие преобразования в формуле (10), получим, что $R_{c}=3.344 \cdot 10^{-10} \mathrm{~m}$ (или $3.34 \AA$ ). Это значение близко к значению расстояния межмолекулярных связей в воде, например, измеренное расстояние между соседними атомами кислорода (О-O) варьируется от 0.27 до $0.3 \mathrm{~nm}$ [46-48], а между кислородом и водородом $(\mathrm{H}-\mathrm{O})$ размер от 0.096 до $0.1 \mathrm{~nm}[48,49]$. Таким образом, можно предположить, что энергия $G$ в формуле (6) относится к энергии зародышеобразования на элементарном межмолекулярном уровне. Данный результат требует дальнейшего исследования. Однако он демонстрирует физическую суть инкубационного времени кавитации по формуле (6).

Дополнительно отметим, что в теории нуклеации установлена связь между числом событий нуклеации, происходящих в единице объема в единицу времени, т.е. скоростью нуклеации $J$ и числом Гиббса $G b$, которая может быть представлена в общем виде как

$$
J=J_{0} e^{\frac{-W_{c r}}{k T}},
$$

где $J_{0}$ - некоторый коэффициент пропорциональности. Тогда, рассматривая систему уравнений из (6) и (11), можно получить соотношение для характеристик теории нуклеации и критерия инкубационного времени кавитации

$$
\frac{J}{J_{0}}=\frac{\tau_{0}}{\tau}=\frac{1}{e^{G b}}
$$

Соотношение (12) открывает новые возможности для исследования природы инкубационного времени кавитации или фазовых переходов в рамках структурновременного подхода [21,27,34].

Таким образом, несмотря на то, что параметры $\tau$ и $\alpha$ пока не могут быть определены каким-либо прямым расчетом или экспериментом по кавитации, они потенциально могут рассматриваться как физические величины. Этот вопрос требует дополнительных исследований. Однако на основе представленных результатов можно заключить, что данные параметры позволяют косвенным образом учитывать влияние скорости энергетических процессов, преодоление вязкости и инерционных процессов на пороговые значения начала кавитации жидкости при различных частотах ультразвука, температуры жидкости и гиростатического давления.

\section{Заключение}

В работе представлен анализ изменения пороговых характеристик акустической кавитации жидкости на примере воды в зависимости от частоты колебаний звукового поля, гидростатического давления и температуры жидкости. Расчет данных зависимостей проводился на основе критерия инкубационного времени кавитации и классического критерия порога нестабильной кавитации. Первый критерий использует только макропараметры жидкости, а для применения второго критерия необходимо учитывать размеры кавитационных микропузырьков. Таким образом, проведено сравнение двух подходов к оценке прочности жидкости при динамических воздействиях. В качестве основных результатов работы можно выделить следующее.

Показана возможность применения критерия инкубационного времени для моделирования порогового давления начала кавитации в широком диапазоне частот ультразвука, при различных температурах жидкости и гидростатических давлениях. При этом критерий позволяет проводить анализ порогового давления нестабильной кавитации без прямого учета параметров дефектной микроструктуры жидкости. В свою очередь классический критерий порога нестабильной кавитации не позволяет качественно моделировать рассмотренные зависимости.

Получено, что комбинация двух подходов позволяет определить диапазон радиусов зародышей, которые могут привести к нестабильной кавитации, в зависимости от частоты звуковых колебаний в жидкости. Поскольку каждой частоте колебаний соответствует своя пороговая амплитуда колебаний, за счет вариации частоты и/или амплитуды звуковых колебаний возможно установить 
оптимальный режим для начала кавитации жидкости, в том числе с учетом заданного размера зародышей кавитации. В дальнейшем такая комбинация двух критериев может быть использована для установления связи микро- и макропараметров прочности жидкости.

Показано, что характеристики прочности жидкости, используемые в критерии инкубационного времени, могут быть связаны с характеристиками вязкости жидкости, энергии образования зародыша кавитации, а также скорости нуклеации зародышей. Данная связь может быть использована в дальнейшем для прямого расчета параметров критерия инкубационного времени кавитации.

Поскольку экспериментальные данные по зависимости порога начала кавитации от рассмотренных условий воздействия весьма ограничены, одним из дальнейших направлений развития представленных результатов является постановка и проведение соответствующих корректных экспериментов. Не менее важным развитием результатов является оценка влияния характеристик вязкости и инерции жидкости на рассмотренные зависимости. Кроме того, в критерии инкубационного времени кавитации необходимо предусмотреть учет вероятностных характеристик случайных процессов зарождения кавитации.

Таким образом, несмотря на необходимость дальнейшего развития подхода инкубационного времени, результаты работы демонстрируют его преимущество перед классическим критерием порога кавитации. По аналогии с динамическим разрушением твердых тел учет соотношения характерного инкубационного времени начала кавитации в жидкости с длительностью, и тем самым скоростью подвода энергии в область кавитации позволяет качественно моделировать начало кавитации в зависимости от широкого набора внешних условий воздействия.

\section{Финансирование работы}

Работа поддержана грантом Президента Российской Федерации для молодых ученых (МК-2269.2019.8). Разд. 2 подготовлен по результатам исследований в рамках гранта Российского научного фонда (грант № 2079-10078).

\section{Конфликт интересов}

Авторы заявляют, что у них нет конфликта интересов.

\section{Список литературы}

[1] Физика и техника мощного ультразвука. Мощные ультразвуковые поля, под. ред. Л.Д. Розенберга (Наука, M., 1968), т. 2. [High-Intensity Ultrasonic Fields, Ed. L.D. Rozenberg (Springer US, Boston, 1971)]

[2] C.E. Brennen. Cavitation and Bubble Dynamics (Oxford University Press, NY., 1995)
[3] J. Dai, M. Bai, C. Li, H. Cui, L. Lin. Trends Food Sci. Technol., 105, 211 (2020). https://doi.org/10.1016/j.tifs.2020.09.016

[4] M.B. Mane, V.M. Bhandari, K. Balapure, V.V. Ranade. Ultrason. Sonochem., 61, 104820 (2020). https://doi.org/10.1016/j.ultsonch.2019.104820

[5] Г.Л. Шарипов, А.М. Абдрахманов, Б.М. Гареев. ЖТФ, 83 (2), 107 (2013). https://journals.ioffe.ru/articles/10828 [G.L. Sharipov, A.M. Abdrakhmanov, B.M. Gareev. Tech. Phys., 58 (2), 255 (2013). https://doi.org/10.1134/S1063784213020217]

[6] S.K. Bhangu, M. Ashokkumar. Top. Curr. Chem., 374, 56 (2016). https://doi.org/10.1007/s41061-016-0054-y

[7] M. Zupanc, Ž. Pandur, T. Stepišnik Perdih, D. Stopar, M. Petkovšek, M. Dular. Ultrason. Sonochem., 57, 147 (2019). https://doi.org/10.1016/j.ultsonch.2019.05.009

[8] B. Dollet, P. Marmottant, V. Garbin. Annu. Rev. Fluid. Mech., 51, 331 (2019). https://doi.org/10.1146/annurev-fluid-010518-040352

[9] C.E.H. Tonry, V. Bojarevics, G. Djambazov, K. Pericleous. JOM, 72, 4082 (2020). https://doi.org/10.1007/s11837-020-04370-7

[10] A. Priyadarshi, M. Khavari, T. Subroto, M. Conte, P. Prentice, K. Pericleous, D. Eskin, J. Durodola, I. Tzanakis. Ultrason. Sonochem., 70, 105260 (2021). https://doi.org/10.1016/j.ultsonch.2020.105260

[11] K.J. Pahk, S. Lee, P. Gélat, M.O. de Andrade, N. Saffari. Ultrason. Sonochem., 70, 105312 (2021). https://doi.org/10.1016/j.ultsonch.2020.105312

[12] E. Stride, T. Segers, G. Lajoinie, S. Cherkaoui, T. Bettinger, M. Versluis, M. Borden. Ultrasound Med. Biol., 46, 1326 (2020). https://doi.org/10.1016/j.ultrasmedbio.2020.01.027

[13] S. Nalesso, M.J. Bussemaker, R.P. Sear, M. Hodnett, J. Lee. Ultrason. Sonochem., 57, 125 (2019). https://doi.org/10.1016/j.ultsonch.2019.04.020

[14] V.G. Baidakov, A.M. Kaverin. Int. J. Heat. Mass. Transf., 163, 120498 (2020). https://doi.org/10.1016/j.ijheatmasstransfer.2020.120498

[15] F. Caupin. J. Non Cryst. Solids, 407, 441 (2015). https://doi.org/10.1016/j.jnoncrysol.2014.09.037

[16] Н.В. Малых, Г.Н. Санкин. ЖТФ, 80 (1), 92 (2010). https://journals.ioffe.ru/articles/9897 [N.V. Malykh, G.N. Sankin. Tech. Phys., 55 (1), 92 (2010). https://doi.org/10.1134/S1063784210010159]

[17] X. Yao, Z. Li, L. Sun, H. Lu. Phys. Fluids, 32, 112019 (2020). https://doi.org/10.1063/5.0026361

[18] R.E. Apfel. Meth. Exp. Phys., 19, 355 (1981). https://doi.org/10.1016/S0076-695X(08)60338-5.

[19] E.A. Neppiras. Ultrasonics, 18, 201 (1980). https://doi.org/10.1016/0041-624X(80)90120-1

[20] А.С. Бесов, В.К. Кедринский, Н.Ф. Морозов, Ю.В. Петров, А.А. Уткин. ДАН, 378 (3), 235 (2001). [A.S. Besov, V.K. Kedrinskii, N.F. Morozov, Y.V. Petrov, A.A. Utkin. Dokl. Phys., 46, 363 (2001). https://doi.org/10.1134/1.1378105]

[21] Ю.В. Петров. ДАН, 395 (5), 621 (2004). [Y.V. Petrov. Dok1. Phys. 49, 246 (2004). https://doi.org/10.1134/1.1753621]

[22] А.А. Груздков, Ю.В. Петров. ЖТФ, 78 (3), 6 (2008). http://journals.ioffe.ru/articles/9355 [A.A. Gruzdkov, Y.V. Petrov. Tech. Phys. 53 (3), 291 (2008).

https://doi.org/10.1134/S106378420803002X] 
[23] А.Д. Евстифеев, А.А. Груздков, Ю.В. Петров. ЖТФ, 88 (7), 59 (2013). http://journals.ioffe.ru/articles/10950 [A.D. Evstifeev, A.A. Gruzdkov, Y.V. Petrov. Tech. Phys., 58 (7), 989 (2013). https://doi.org/10.1134/S1063784213070086]

[24] Ю.В. Петров, А.А. Груздков, В.А. Братов. Физическая мезомеханика, 15 (2), 15 (2012).

http://www.ispms.ru/ru/journals/394/1826 [Y.V. Petrov, A.A. Gruzdkov, V.A. Bratov. Phys. Mesomech., 15, 232 (2012). https://doi.org/10.1134/S1029959912020117]

[25] I. Smirnov, N. Kazarinov, Y. Petrov. Theor. Appl. Fract. Mech., 101, 53 (2019). https://doi.org/10.1016/j.tafmec.2019.02.006

[26] N. Selyutina. Mech. Mater., 150, 103589 (2020). https://doi.org/10.1016/j.mechmat.2020.103589

[27] Г.А. Волков, Ю.В. Петров, А.А. Груздков. ЖТФ, 85 (5), 123 (2015). http://journals.ioffe.ru/articles/41750

G.A. Volkov, Y.V. Petrov, A.A. Gruzdkov. Tech. Phys., 60 (5), 753-756 (2015). https://doi.org/10.1134/S1063784215050278]

[28] B.E. Noltingk, E.A. Neppiras. Proc. Phys. Soc. Sect. B, 63, 674 (1950). https://doi.org/10.1088/0370-1301/63/9/305

[29] R. Esche. Acta. Acust. United with Acust., 2, 208 (1952).

[30] M. Minnaert. XVI. London, Edinburgh, Dublin Philos. Mag. J. Sci., 16 (104), 235 (1933). https://doi.org/10.1080/14786443309462277

[31] V.K. Kedrinskii. Acta Astronaut., 3, 623 (1976). https://doi.org/10.1016/0094-5765(76)90166-1

[32] В.К. Кедринский. ПМТФ, 3, 74 (1993). https://sibran.ru/ journals/issue.php?ID=119948\&ARTICLE_ID=133688

[V.K. Kedrinskii. J. Appl. Mech. Tech. Phys., 34, 361 (1993). https://doi.org/10.1007/BF00864788]

[33] Г.А. Волков, А.А. Груздков, Ю.В. Петров. Физическая акустика, 53 (2), 149 (2007).

http://www.akzh.ru/pdf/2007_2_149-152.pdf [G.A. Volkov, A.A. Gruzdkov, Y.V. Petrov. Acoust. Phys., 53, 119 (2007). https://doi.org/10.1134/S1063771007020017]

[34] Г.А. Волков, А.А. Груздков, Ю.В. Петров. ЖТФ, 79 (11), 147 (2009). https://journals.ioffe.ru/articles/9857 [G.A. Volkov, A.A. Gruzdkov, Y.V. Petrov. Tech. Phys., 54 (11), 1708 (2009). https://doi.org/10.1134/S1063784209110267]

[35] J. Sponer. Czechoslov. J. Phys., 40, 1123 (1990). https://doi.org/10.1007/BF01597973.

[36] T. Thanh Nguyen, Y. Asakura, S. Koda, K. Yasuda. Ultrason. Sonochem., 39, 301 (2017). https://doi.org/10.1016/j.ultsonch.2017.04.037

[37] G.S.B. Lebon, I. Tzanakis, G. Djambazov, K. Pericleous, D.G. Eskin. Ultrason. Sonochem., 37, 660 (2017). https://doi.org/10.1016/j.ultsonch.2017.02.031

[38] A. Žnidarčič, R. Mettin, M. Dular. Ultrason. Sonochem., 22, 482 (2015). https://doi.org/10.1016/j.ultsonch.2014.05.011

[39] Y. Fang, T. Yamamoto, S. Komarov. Ultrason. Sonochem., 48, 79 (2018). https://doi.org/10.1016/j.ultsonch.2018.05.011

[40] G.L. Chahine, A. Kapahi, J.-K. Choi, C.-T. Hsiao. Ultrason. Sonochem., 29, 528 (2016). https://doi.org/10.1016/j.ultsonch.2015.04.026

[41] U. Parlitz, C. Scheffczyk, I. Akhatov, W. Lauterborn. Chaos, Solitons Fractals, 5, 1881-1891 (1995). https://doi.org/10.1016/0960-0779(94)00192-S

[42] A. Brotchie, F. Grieser, M. Ashokkumar. Phys. Rev. Lett., 102, 084302 (2009).

https://doi.org/10.1103/PhysRevLett.102.084302
[43] K.B. Bader, J.L. Raymond, J. Mobley, C.C. Church, D. Felipe Gaitan. J. Acoust. Soc. Am., 132, 728 (2012). https://doi.org/10.1121/1.4733539

[44] S. Palit. Nature, 177, 1180 (1956).

[45] V. Skripov, V. Baidakov, A. Kaverin. Phys. A Stat. Mech. Its Appl., 95, 169 (1979). https://doi.org/10.1016/0378-4371(79)90049-9

[46] K. Liu, J.D. Cruzan, R.J. Saykally. Science, 271, 929 (1996). https://doi.org/10.1126/science.271.5251.929

[47] L. Fu, A. Bienenstock, S. Brennan. J. Chem. Phys., 131, 234702 (2009). https://doi.org/10.1063/1.3273874

[48] A.H. Narten, W.E. Thiessen, L. Blum. Science, 217, 1033 (1982). https://doi.org/10.1126/science.217.4564.1033

[49] M. Hakala, K. Nygård, S. Manninen, L.G.M. Pettersson, K. Hämäläinen. Phys. Rev. B, 73, 035432 (2006). https://doi.org/10.1103/PhysRevB.73.035432 